\title{
Population Behaviors in Crisis Situations - A Study of Behavioral Factors in the PPI Ineos Emergency Response Exercise
}

\author{
Maude Arru \\ Paris-Dauphine University \\ PSL Research Universities \\ CNRS, LAMSADE \\ 75016 Paris, France \\ maude.arru@dauphine.fr
}

\author{
Elsa Negre \\ Paris-Dauphine University \\ PSL Research Universities \\ CNRS, LAMSADE \\ 75016 Paris, France \\ elsa.negre@dauphine.fr
}

\author{
Camille Rosenthal-Sabroux \\ Paris-Dauphine University \\ PSL Research Universities \\ CNRS, LAMSADE \\ 75016 Paris, France \\ camille.rosenthal- \\ sabroux@dauphine.fr
}

\begin{abstract}
Warnings can help to prevent damage and harm if they are issued timely and provide information that helps respondents and population to adequately prepare for the disaster to come. Today, many indicators and sensor systems are designed to produce alert and reduce disaster risks. These systems have proved to be effective but, as all systems including human beings, part of the system remains unpredictable. Each person behaves differently when a problem arises.

We study in this paper the reactions of the population of Verdun, in France, during a public safety exercise. This exercise simulated a chemical risk alert, including the population participation. We propose here an analysis of people's reactions during this exercise, based on interviews and surveys, and according to different behavioral factors.
\end{abstract}

\section{Introduction}

Today, thanks to Information and Communication Technologies (ICT), it is faster and more efficient to manage real time data, make maps from geolocalized data or make assessments based on scenarios that integrate data from different sources. These evolutions enabled to improve crisis management systems, developed to support those who respond to disasters. These crisis management systems help in particular to predict as precisely and as soon as possible the consequences of a crisis and its evolution in a given territory. Despite knowledge and technologies developed in order to minimize or avoid disastrous consequences that a crisis can produce, they remain, by definition, determined by uncertain phenomena, which are not always considered in these crisis management systems. The vulnerability of territories, the need for coordination among services, and the probable behaviors of populations-in-danger, for example, are sometimes neglected.

Before and after a crisis, people act according to their own knowledge and interpretation schemes. These schemes do not always allow people to react in an appropriate way to risky situations and can lead to dangerous reactions. To respond to these problems, we propose to add concerns about human behaviors to the design methodologies of Early-Warning Systems. The behavior concept needs to be clarified and well defined, since it can be approached very differently in the scientific sphere. Some speak of "nomadic" concept that can take on several meanings according to the disciplines [1]. In philosophy, for example, definitions rest on the notions of conscience and experiences [2], although in cognitive sciences it can be approached as a logical suite of actions [3]. The most important works on the subject are provided by human sciences, notably in ethology and in psychology domains $[4,5]$. In this paper we take up the definition of [6] for whom the behavior corresponds to the "reactions of a person, considered in a milieu and in a given time unit to an excitation or a set of stimulation". Human behavior is also integrated in artificial intelligence research whose idea is to transport knowledge elements in a virtual reality and to provide reasoning for the treatment of these elements. Applications of artificial intelligence enable virtual agents to make strategic choices. We find these kinds of research in domains such as automatic production of explanations or solving mathematical problems [7], but it is still difficult today to integrate cognitive dimensions of behaviors to these computer science representations. Individual behaviors in crisis situations do not correspond to everyday life behaviors. It is difficult to represent these behaviors from the information that have been obtained after a crisis, as this information is always static, punctual and contextual. This causes difficulties to integrate the 
great diversity of human reactions that can appear in crisis situations. We can however work to establish tendencies, or correlations on factors that orient particular behaviors. Understanding human behavior in a way that could be integrated in intelligent systems is still a challenge that needs the interconnection of heterogeneous elements such as personality, social or environmental items, logic.

Warning Systems are key elements in crisis management systems. They allow people to orient their behavior when a crisis is announced by providing them information before the crisis, and orienting them in the interpretation of the signals perceived during the crisis. ICT are particularly used in these systems but the potential influence of communication is sometimes under-exploited.

The consideration of laws and phenomena that influence human behaviors in crisis situations seems for us an important area of research and reflection on the improvement of these Warning Systems: warning broadcasts, crisis communication, and the development of educational policies and targeted awareness. The behavior of a population faced with a disaster is not the sum of individual's behaviors. Each person has his own interpretative framework, but an objective for crisis managers is to lead people to a common knowledge and reaction that can be integrated in individual interpretative framework. In this paper, we propose to study people's reactions during a public safety exercise organized in the city of Verdun, in France. Our objective is to lead to take into account the result of the analysis of behavioral factors in the design of an Early-Warning System.

The structure of the paper is as follows. In the next section, we propose to present what are Early-Warning Systems and the context of our study, we then outline the method used to analyze people's reactions during this exercise, before discussing the outcome. Finally we present research perspectives and future work.

\section{Early-Warning Systems}

Early-warning is the provision of timely and effective information that allows organizations and individuals to take action to avoid or reduce their risk and prepare for effective response [8]. According to the nature of disaster, Warning Systems (WS) or Early-Warning Systems (EWS) are provided, WS being limited to unpredictable disasters, in conditions where no signals can be interpreted in advance to assess the potential disasters and prepare populations. In the case of snowfall for example, conditions that cause disasters are so complex and dependent on each other that experts can only draw up risk maps or organize planned prevention actions and alert populations when events occur, effectively. They cannot assess the event and evacuate the population in advance. This is the main difference between WS and EWS which provide warnings with sufficient time for people to act.

We consider more precisely in this paper, according to [9], that an EWS is a "Chain of information communication systems comprising sensor, detection, decision, and broker-subsystems, in the given order, working in conjunction, forecasting and signaling disturbances adversely acting the stability of the physical world; and giving sufficient time for the response system to prepare resources and response actions to minimize the impact on the stability of the physical world".

A complete and effective EWS comprises four elements [10]:

- Risk knowledge: knowledge of the relevant hazard and vulnerability;

- Monitoring and warning service: technical capacities to constantly monitor hazard precursors, prediction of potential risks and warning issue;

- Dissemination and communication: dissemination of understandable warnings with prior preparedness information;

- Response capability: knowledge of risks, warning services plans and appropriate actions for persons at risk.

In this sequential list, each element has two direct links and interactions with each of the other elements. Failure of any part of the system will imply failure of the whole system. Human factor in particular plays a significant and transversal role in all steps $[11,12]$.

Before and during disasters, people often act according to their own interpretation schemes [13] which are not always adapted to risk situations and can lead to dangerous reactions. Communication technology is a key element in EWS to improve behaviors, it provides common pre-disaster knowledge before disasters, and guidance to interpret cues during events. The potential influence of communication can be under exploited, for example, warnings are often simplified and reduced to a simple "Red Alert", instead of providing concrete advice and guidance [14].

EWS are constantly evolving, reliability indicators and modelling methods such as Bayesian Networks $[15,16,17]$ permit us to evaluate their technical quality and performance. These indicators are objective and useful to make decision-makers aware, capable of taking measures for improvement or reorganization of the system's materials. They do not 
allow to evaluate the human elements of EWS, especially response capability. Risk comprehension and interpretation of warnings is highly subjective but they can have important consequences such as the inappropriate responses to warnings delivered by Météo-France $^{1}$ in October 2015 that have not been followed and lead to the deaths of people trapped in their cars in tunnels or in underground car parks, as they tried to get their cars out.

\section{Context}

To test the interest of integrating behavioral studies to EWS, we plan to analyze populations behaviors in reaction to warnings triggered during several exercises organized in France.

\subsection{Public safety in France}

In France, protection against accidents and disasters is a function of the State. This role is provided by the civil safety teams, which rests on different specialist services who act for civil safety, firefighters, military units of training and intervention, pilots of aircraft and helicopters as well as mineclearing experts. Their roles are directed by the Direction Générale de la Sécurité Civile et de la Gestion des Crises (General Direction of the Civil Safety and of the Crisis Management) under the direction of the Ministry of the Interior. They define particularly the roles of evaluation, preparation, coordination and application of protection, the information and Warning Systems for populations, the prevention of civil risks of all types, and the planning of civil security measures. This organization rests on the 101 prefectures present on the French territory.

In this study, we propose to analyze people's reactions to warnings tested during a civil safety exercise organized by the prefecture of Verdun ${ }^{2}$. This exercise responds to a European Directive that imposes all SEVESO industrials with a high risk of accident to organize every three years a civil safety exercise. Industries classified as SEVESO sites are establishments where the quantity of dangerous products exceeds the thresholds laid down in the European SEVESO Directive. In accordance with the Directive, the French regulatory texts require the different actors to draw up action plans which have been tested during the exercise in Verdun:

\footnotetext{
${ }^{1}$ Météo-France is the French national meteorological service.

${ }^{2}$ It is a small city of the Meuse department in Grand

Est region, located in the northeastern France. Its area
}

- $\quad$ the PPI (Particular Plan of Intervention), managed by the prefecture;

- $\quad$ the POI (Internal Operation Plan), managed by the company;

- $\quad$ the PCS (Communal Plan of Protection) managed by the municipalities.

These exercises can also implicate population if the civil safety teams in the prefectures decide to test the population participation. In France, for some years, the number of exercises that include the population participation has significantly increased.

\subsection{PPI Inéos exercise}

The PPI Inéos exercise, realized on March 22 2017 , was dedicated to test the actions plans of the different actors, the communication between the actors, the public information management and the Warning System in response to an accident that would happen at the Inéos company, which would lead to the liberation of a toxic cloud of chlorine.

It was prepared with the cooperation of the Inéos company, the local collectivities, including twelve municipalities that participated to the exercise, the regional roads management, the meteorology services, the rescue services and the telecom, electricity and gas organizations.

In this study, we focus on the populations reactions to the warnings that have been triggered. Two kinds of warning were triggered by the state services: the sirens of two different municipalities and messages provided by the smartphone application SAIP (Alert and Information System for the Population). This application send warning messages to people who have already installed the application, it requires an internet connection to be active. Some others warnings were deployed by municipalities such as alarm bells or interphone communications.

A campaign of awareness had been launched before the exercise to inform people of the date of the exercise and to specify the waited reactions. When the warnings were triggered, people had to react according to their situation.

- If they were outside, they had to move to the closest building, the street being the more exposed area.

- If they were traveling by car, they had to cut the ventilation of the vehicle, stop and to take refuge in the closest building. They should not try to escape. Escape in the situation simulated during

is $31.03 \mathrm{~km}^{2}(11.98 \mathrm{sq} \mathrm{mi})$ with a density of $590 / \mathrm{km}^{2}$ (227,8/sq mi). 18,327 inhabitants live in the city. 
the exercise was dangerous for their own safety and disturbing for the circulation of the responding emergency services vehicles.

- If they were inside, people had to stay inside the building. They should not go towards the incident or try to collect their children from school.

Once in a safe place, they had to close doors, windows and draughts, stop air-cons and reduce the heating. They had to breathe through a humid cloth, listen to information from local Media and keep calm waiting for the warning signal for the end of the exercise or an evacuation order.

The Warning System tested in the PPI Inéos can be, to a certain extent, considered as an EWS. Indeed, unlike wildfires, hurricanes, earthquakes or even financial or ecological crisis that can be detected in advance, the industrial risk of liberation of a toxic cloud of chlorine cannot be predicted, but the crisis itself can be prepared for and anticipated according to the cloud movements.

\section{Method and panel}

\subsection{Behavioral factors studied}

In a previous study [18], we identified twenty factors that influence human behaviors in crisis situations from researches in literature. We assembled them in two categories according to the objects they refer to, individual and environmental, and to the types of information sources that allow to characterize them. We present here these factors with a list of indicators associated to each of them that have been studied in the PPI Inéos exercise. We did not collect information on all of the indicators that are listed for these factors.

- Civil status: age, sex, nationality, residence place, level of schooling, occupation.

- Personality: desires, moral principles, sociability, beliefs, religious or not, capacity to take decisions, mimetic reactivity.

- Motivation to escape/defend: motivations are strongly related with the experience, risk assessment, the current action and the physiological signals identified after.

- Responsibility: in the situation when a fire alert is given in a school for example, a teacher can have different reactions depending on whether he is alone in his office or he is in a classroom, teaching to a student group. He may have no reaction if he is alone, but he will be much well-disposed to evacuate with his students in good conditions if he is responsible for them and has to set an example for them to follow.
- Emotions: joy, sadness, anger, disgust, fear, surprise, contempt.

- Experience: crisis faced in the past, objective ability to escape/defend, subjective ability to escape/defend.

- $\quad$ Explicited knowledge: general knowledge shared by the population, training followed, access to documents, access to knowledge sharing tools [19].

- Risk assessment: objective assessment, subjective assessment.

- Perception of the EWS: we define perception as the process of collecting, organizing, and interpreting stimuli which may be information or knowledge coming from different sources [20].

- Current action: interaction, concentration and movement needed.

- Geographic zone characteristics [21]: zone extent, population density, poverty level, economic status, urban level, population pyramid, cultural characteristics: individual versus group orientation, trust in government.

- Interaction and mobility capacity: frequented area, smart-phone, access to transport.

- Perceptible signals of the crisis: indicators of perceptible signals depend directly of the type of crisis that is concerned. This is why we have defined three generic indicators: visual signals, sound signals, and olfactory signals.

- Period characteristics: day/night, number of hours from the crisis peak.

- Alerts / Transmitted information: quantity and quality of information transmitted, number of broadcasting channels [22].

- Entourage characteristics: density of population, presence of authority representatives, security level of the area, presence of close relations.

- Behaviors of the closest people: contagion level of the three dominant behaviors. The contagion level of an agent varies according to the number of neighborhoods with similar activities [23, 24].

Data on the different factors have been collected from questions asked to the population on the area of the PPI Inéos exercise and from information on the places where people have been questioned. One of our objectives was to obtain different perspectives to understand people's reactions during the exercise. This is why we used a survey largely open, with only few closed questions, to enhance our understanding and let people think about their reactions and the goal of the exercise. 


\subsection{Data collection}

\subsubsection{The survey}

Interviews have been conducted from face-to-face meetings and online surveys, we also launched an online anonymous survey in March 2017, created with the Open Source software survey tool Limesurvey ${ }^{3}$. We collected information about the age, sex, profession and place of residence of the participants. Then, they answered to four sets of questions.

The first set focused on people's knowledge of the exercise: awareness to recognize warnings, training, information received about risks and waited behaviors, and risk perception when a warning is triggered. This set included also questions about the actions that people were doing when the warnings were launched.

The second set of questions referred to people's perception of warnings, their understanding and comments on the siren and the message sent by the SAIP application for smartphone (if they had downloaded it before).

Then, a third set of questions addressed only to people who received warnings, directly or not were related to the feelings and actions of these people during the exercise. We asked questions about their emotions, stress levels, time between the warning trigger and their actions, their reflection on the possibility of taking other actions, their communication during the exercise, the reactions of people around them and their judgment on these reactions. We also asked questions about leadership phenomenon.

Finally, the fourth set was related to the experiences of people in similar crisis, their reflections about the possible reactions they may have in risky situations that are not exercises, and information about their personality.

\subsubsection{Data analysis}

For this study, 134 people were questioned. 19 people answered the paper survey (from face-to-face meetings) on the day of the exercise, we conducted these interviews in and around the area of the St Anne High School, in the city of Verdun. 115 people replied to the online survey, during the days that followed the exercise. On these 115 responses, 38 come from the online version of the survey that the Town Halls sent to their residents, and the last 77 replies come from an online adapted version of the survey made for the students and teachers of the St Anne High School.

\section{Results and discussion}

\subsection{About the respondents}

Figure 1 shows the distribution of respondents by age: about $43 \%$ are between the ages of 16 and 25, which can be explained by the proximity of the person interviewing the respondents to a school.

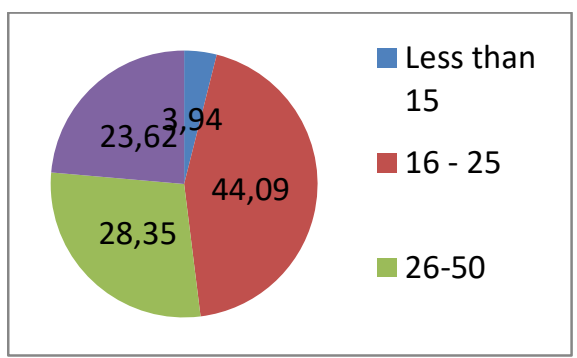

Figure 1: Age of respondents (in \%)

Moreover, among the 44 people who agreed to give information about their job, it appears in Figure 2 that in addition to students, many retirees and employees responded to the survey. Many socioprofessional categories feel concerned.

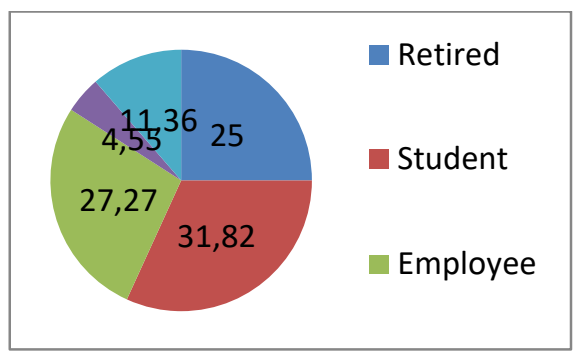

Figure 2: Socio-Professional category of respondents (in \%)

As displayed on Figure 3, among the 46 people who agreed to indicate their place of residence, we can observe that about $37 \%$ of respondents lived in Verdun or its suburbs and $39 \%$ within $5 \mathrm{~km}$ around Verdun. Only citizens located near the place of the exercise answered to the survey.

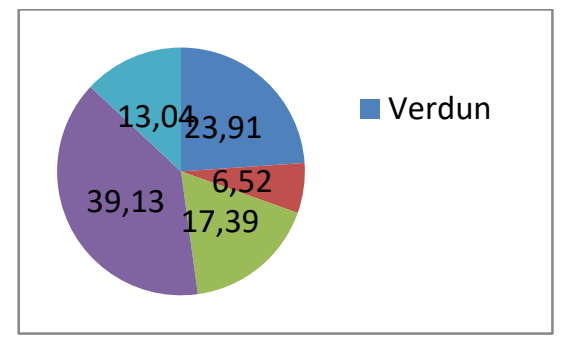

Figure 3: Place of residence of respondents (in \%)

\footnotetext{
${ }^{3}$ https://www.limesurvey.org
} 
Finally, as displayed on Figure 4, the three main personality traits that correspond to the respondents are: calm (about 52\%), rational (about 35\%) and generous (about 27\%).

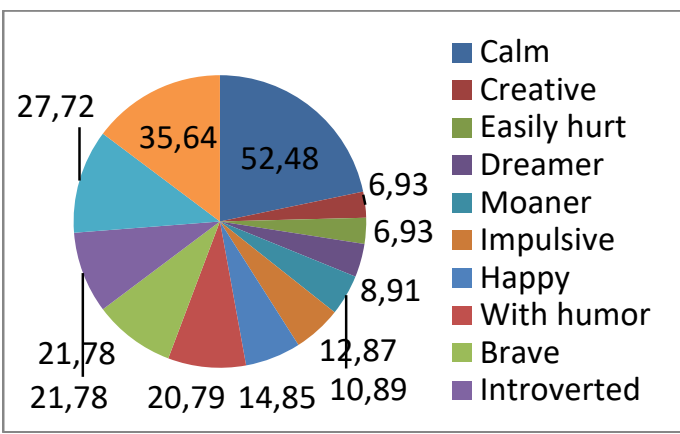

Figure 4: Representative personality traits (> 5\% of the population)

\subsection{Awareness / Preparation}

The majority of respondents were aware of the alert and they knew the behavior to adopt through poster campaign or by the practice of past exercises. Although the efforts of the mayors on the exercise were highlighted, some respondents made remarks about raising awareness. In particular, they talked about the need to do exercises more frequently, to reinforce the poster campaign, to better inform the population by indicating which signal corresponds to what risk and finally to raise the sound level of the siren which was not heard in some cities located at more than $5 \mathrm{~km}$ (3.1 miles) of the siren.

We can note that respondents are aware of their level of risk exposure when the alert is triggered outside an exercise: as displayed on Figure 5, more than $40 \%$ consider that there is a risk and more than $49 \%$ consider that this risk is large or huge.

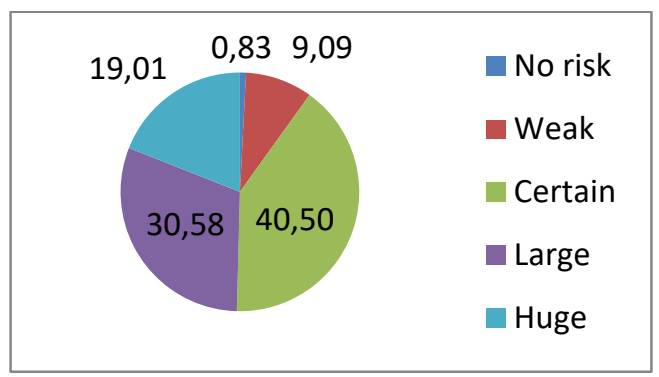

Figure 5: Level of risk exposure when the alert is triggered outside an exercise (perceived by respondents - in \%)

We discovered an association of these levels with age of respondents, as showed on Figure 6, only a fraction of those over 15 consider the risk to be huge, the proportion being highest amongst those of 50 years or older, with $25 \%$ of them considering that the risk is huge when they hear the warnings outside an exercise. It is finally noteworthy that $20 \%$ of under 15 year olds consider that the risk is low.

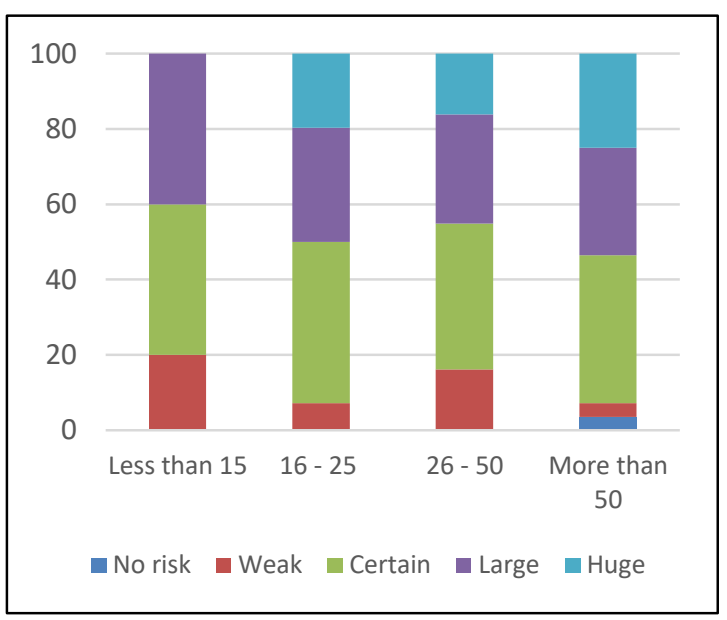

Figure 6: Level of risk exposure when the alert is triggered outside an exercise according to the age of the respondents (perceived by respondents - in \%)

\subsection{At the time of the alert}

$77 \%$ of respondents heard the siren. As mentioned earlier, this can be explained by the distance between the place where the alert have been triggered and the location of the respondents. In addition, some people indicated that they did not pay attention to the signals. Around 18\% did not understand that it was an alert, and, unfortunately, $90 \%$ of the respondents did not receive the message from the SAIP (Alert and Information System for the Population) application on their smartphone. However, as shown in Figure 7, respondents were predominantly at work or at school: about $67 \%$ (this figure is biased because interviews were conducted near a school), or outside. Preparation in the workplace / school seems to be essential.

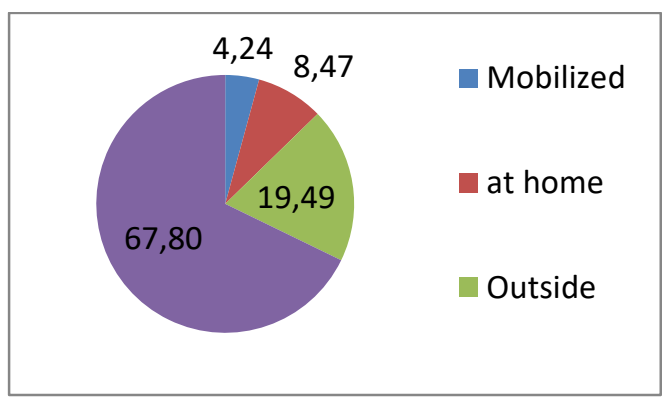

Figure 7: Activity when triggering the alert (in \%) 
Indeed, risk culture is particularly important in the St Anne High School of Verdun. Every year the school organizes exercises for fire safety or attack simulations. The latest exercise which simulated an attack had only been realized one month before the PPI Inéos exercise. Teachers were prepared to face a crisis, and were vigilant to warnings. For each exercise, they receive information and instructions from a dedicated application created by the school for crisis response. This application is highly appreciated by teachers, and it's instructions are followed. This is not the case for the national SAIP application for the main reason that the existence of the application is unknown for most of people and that the warnings that were sent by the application were sometimes not received or too late, at least more than thirty minutes after the sirens were activated.

Reactions to warnings are contrasted. Most people who responded to the poll had confined themselves, but many of them replied that they had done nothing, for different reasons: they had not heard the siren (the sound level was too low), they knew "it was a false alarm", they did not see anyone in the city reacting, they could not identify what they had to do and many of them were not sure whether the siren was a warning or only a test of the proper functioning of the equipment.

As shown in Figure 8, the majority of people (22\%) who felt responsible for other people did not do anything to help them. It would be interesting to verify or test this result after real crisis situations.

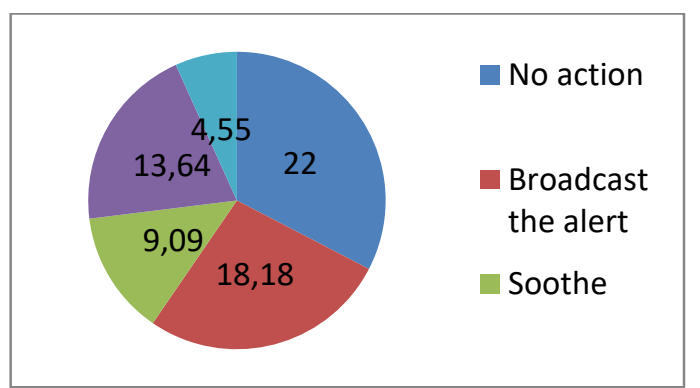

Figure 8: Actions carried out by people who felt responsible (from close or unknown people - in \%)

Crossing the actions of people who felt responsible for others with the main personality traits, we can observe, as shown on Figure 9, that people who consider themselves in solidarity with others, attentive or responsible responded almost in the same proportion that they broadcasted the alert, they soothed or they guided people. During the exercise, voluntary, organized and experiented people did not think to soothe close relations. And finally people who did not have any reactions were mostly calm and less significantly organized people.

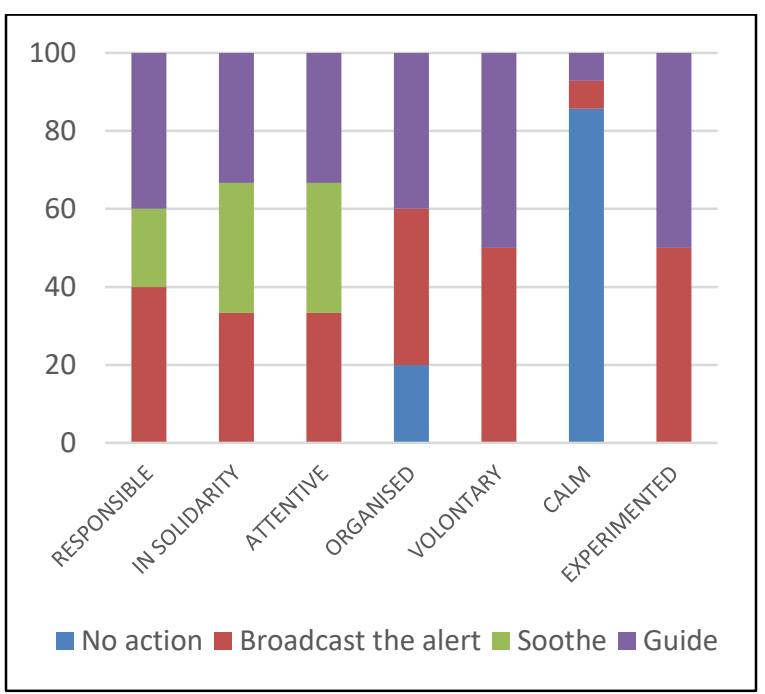

Figure 9: Actions carried out by people who felt responsible according to their personality, for personalities that correspond to actions (from close relations or strangers - in \%)

On Figure 10, we can observe that over $70 \%$ of respondents indicated that they did not feel any emotion at receiving the alert. Only $11 \%$ reported being stressed and 9\% stunned. Of course, these results needs to be put into perspective, most respondents knew that it was an exercise.

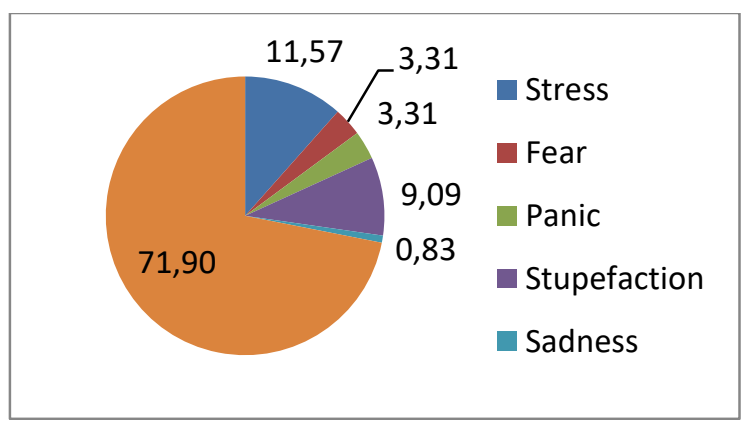

Figure 10: Emotions felt on receiving the alert (in \%)

Figure 11 shows that more than $60 \%$ of respondents acted immediately after the alert and about $20 \%$ in the first half hour. Again, it seems that $14 \%$ of respondents did not play the game (no action). 


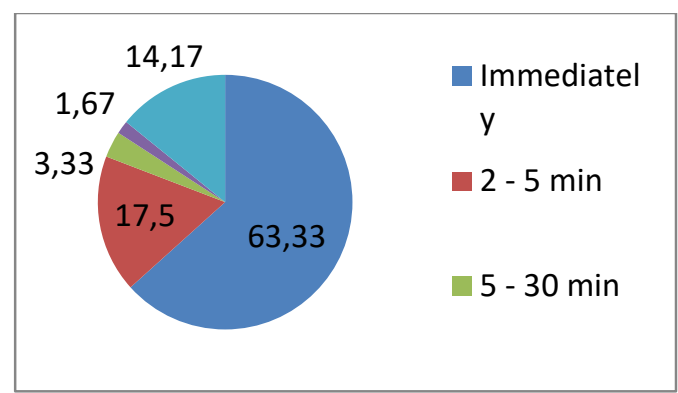

Figure 11: Time elapsed between the triggering of the alert and the actions of the respondents (in \%)

To remain in non-action, $75 \%$ of respondents did not contact relatives. In the majority of cases, respondents indicated that they did not establish contact because they either knew that it was an exercise or they knew that they should not saturate telephone lines.

We can note that only a small number of people of 50 years old or older, when they react, had a time of reaction longer than 30 minutes, see Figure 12. The greater proportion of people who did not react came from the 26 to 50 years of age group. This result reveals a relationship between the age and the activity of people and their time of reaction.

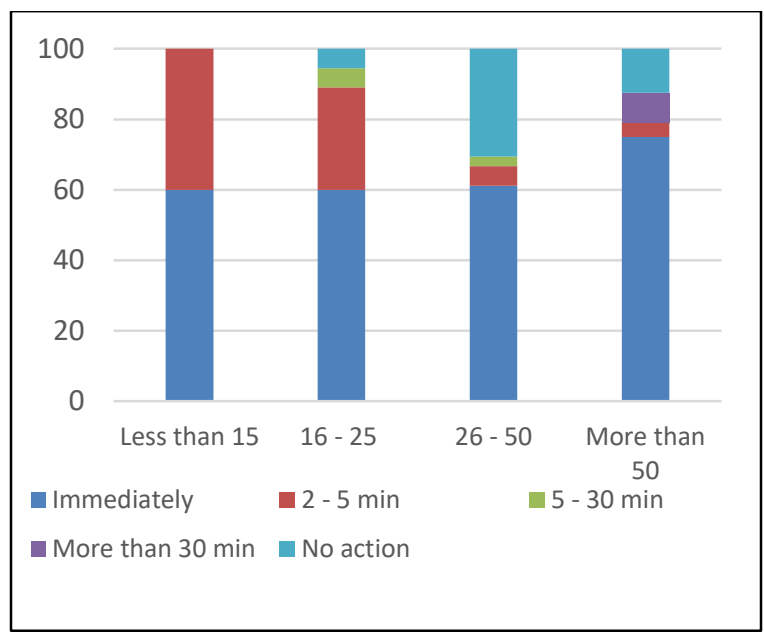

Figure 12: Time elapsed between the triggering of the alert and the actions of the respondents according to their age (in \%)

\subsection{Feedback}

More than $43 \%$ of respondents considered that the people around them showed an appropriate response to the alert. Otherwise, it should be noted that only $15 \%$ of respondents had experienced a similar situation through exercises, of which only $2.5 \%$ had experienced a real situation close to the situation of this exercise. Finally, over $62 \%$ think that they would have reacted differently if they had not known the date of the exercise.

\subsection{Discussion}

The diversity of reactions and perceptions related to the exercise, on risk and warning perception, reaction time, emotions or crisis preparedness engage us to pursue the objective of understanding behavioral factors in crisis situations. We are conscious that the PPI Inéos exercise was not considered as a real crisis for citizens, and reactions were of course different than they would be in a real crisis. Nevertheless, from the interviews with citizens, we consider that people are able to have a constructed and critical opinion on their reactions during the exercise, and are able to project themself in the hypothesis of a real crisis.

Our analysis implicates also various biases. We emphasize the fact that $71 \%$ of respondents were in or around the St Anne High School premises, the population of students from 15 to 18 years old and of teachers are then over-represented. In addition, the support was not completely homogenous, we used paper forms to proceed for the face-to-face interviews which can have an impact on answers, such as the fact that the online survey proposed by the direction of the St Anne High School have been slightly adapted to suit the respondents.

This analysis is specific to the environment where the exercise has been made. Contextual factors in a vulnerability study could complement this survey and give more accurate information to help discover correlations between the different behavioral factors. The density of population for example, the poverty level or the cultural background of the area are indicators that could be considered as step backwards on the results for more generic work.

The objective of integrating our results in the Early-Warning System of the PPI Inéos plan to improve population responses could be realized by different integration levels, according to the four elements of Early-Warning Systems:

- Risk knowledge: for a better field knowledge, notably from morphological indicators;

- Monitoring and warning service: no improvements are considered for this element at this time;

- Dissemination and communication: the vocabulary and the information of warnings and messages could be adapted according to the channel diffusion for a targeted communication;

- Response capability: interviews and online surveys revealed the importance of the exercises 
and of the perception of risks. Training and population awareness could be targeted according to the needs identified. For example, communication could be improved by the use of specific words such as "entertainment" more than "exercise" or "false alarm", such as retained from responses.

\section{Conclusion and Future Work}

In this paper, we study the reactions of the population of Verdun (France) during a public safety exercise (PPI Inéos exercise). This exercise simulated a chemical risk alert. Our analysis of people's reactions during this exercise, based on interviews and surveys highlights some important points. Although many people feel concerned, whatever their age or socio-professional categories, the persons have responded according to the recommended behaviors. It turns out that the siren was not heard/understood by all in the same way or that some people did not play the game. Our analysis shows that some information cannot be retrieved via non-human sensors, and that taking populations into account, especially through their behavior, is of primary importance. Many areas of Early-Warning Systems can be improved, including dissemination and preparedness.

This work lays the theoretical foundations for ongoing work, in which the forms will be extended to incorporate new morphological questions, more precise and accurate scales, and proposition of responses. The free text responses will be reduced and only proposed in the supports for physical interviews. Future work consists also in his conceptual part in the creation of an ontology to provide a clear and shared semantic vision of the concepts related to behaviors and warnings that we use.

Finally, the heterogeneity of the indicators that we presented leads us to adaptations and to propose formulas of aggregation that are not presented here. From these improvements tested in next civil safety exercises, we plan to provide statistical work for data analysis with Multiple Correspondence Analysis methods.

\section{Acknowledgements}

The experimentation has been possible thanks to the help and approval of the planning, exercises and feedback office of the DGSCGC, the civil safety department of the Prefecture of Verdun and thanks to the direction of the St Anne High School of Verdun. We are grateful to all participants of the survey for the time taken to answer our questions and for their useful suggestions.

\section{References}

[1] A.M. Toniolo, "Behavior: From perception to action, a concept to be revisited", In: L'année psychologique, 2009, pp.155-193.

[2] M. Perleau-Ponty, The structure of behavior, Boston: Beacon Press, 1967.

[3] B. F. Skinner, Science and human behavior, Simon and Schuster, 1953.

[4] J. Alcock. Animal behavior. Sinauer Associates Sunderland, 1989.

[5] J. O Heron Cooper et al. Applied behavior analysis. Pearson; $2^{\text {nd }}$ ed., 2007.

[6] N. Sillamy, Dictionary of psychology, Publishing House Encyclopedic Universe, Bucureşti, 1983.

[7] N. Balacheff. "Didactique et intelligence artificielle". In: Recherches en didactique des mathématiques 14, 1994, pp. 9-42.

[8] Hyogo Framework for Action, 2005-2015: Building the resilience of nations and communities to disasters. Extract from the International report of the World Conference on Disaster Reduction, UN ISDR, 2005.

[9] Waidyanatha; "Towards a typology of integrated functional early warning systems". In: International Journal of Computer Science, 2010, pp. 31-51.

[10] Basher, R., 2006, Global Early-Warning systems for natural hazards: systematic and people-centred. Philosophical Transactions of the Royal Society of London A: Mathematical, Physical and Engineering Sciences, 364(1845), 2167-2182.

[11] Sorensen, J. H, 2000, Hazard warning systems: Review of 20 years of progress. Natural Hazards Review, 1(2), 119125 .

[12] Twigg, J, 2003, The human factor in Early-Warnings: risk perception and appropriate communications. In EarlyWarning Systems for Natural Disaster Reduction (pp. 1926). Springer Berlin Heidelberg.

[13] Mileti, D. S., and Sorensen, J. H, 1990, Communication of emergency public warnings: A social science perspective and state-of-the-art assessment (No. ORNL-6609). Oak Ridge National Lab., TN (USA).

[14] Comes, T., Mayag, B., and Negre, E, 2015, Beyond Early: Decision Support for Improved Typhoon Warning Systems. 
[15] Krzysztofowicz, R., Kelly, K. S., and Long, D, 1994, Reliability of flood warning systems. Journal of water resources planning and management, 120(6), 906-926.

[16] Paté-Cornell, M. E, 1986, Warning systems in risk management. Risk analysis, 6(2), 223-234.

[17] Sättele, M., Bründl, M., and Straub, D, 2015, Reliability and effectiveness of Early-Warning systems for natural hazards: Concept and application to debris flow warning. Reliability Engineering \& System Safety, 142, 192-202.

[18] M. Arru, B. Mayag, and E. Negre. "People Behaviors in Crisis Situations: Three Modeling Propositions". In: 13th International Conference on Information Systems for Crisis Response and Management. 2017.

[19] M. Arru et al. "Towards a responsible early-warning system: Knowledge implications in decision support design". In: Research Challenges in Information Science (RCIS), 2016 IEEE Tenth International Conference on. IEEE. 2016, pp. 1-12.

[20] M. Arru, B. Mayag, and E. Negre. "Early-Warning System Perception: a Study on Fire Safety". In: 13th International Conference on Information Systems for Crisis Response and Management. 2016.

[21] T. Comes, E. Negre, and B. Mayag. "Beyond Early: Decision Support for Improved Typhoon Warning Systems". In: 13th International Conference on Information Systems for Crisis Response and Management. 2015.

[22] R.L. Goldstone and M.A. Janssen. "Computational models of collective behavior". In: Trends in cognitive sciences 9.9, 2005, pp. 424-430.

[23] M. Granovetter. "Threshold models of collective behavior". In: American journal of sociology, 1978, pp. 1420-1443.

[24] S. Solomon et al. "Social percolation models". In: Physica A: Statistical Mechanics and its Applications 277.1, 2000, pp. 239-2 